\title{
Comparación entre ensambles de Phyllophaga (Coleoptera: Melolonthidae) del Occidente de Cuba
}

\author{
Annery Serrano ${ }^{1}$, Miguel Ángel Morón ${ }^{1}$, Alejandro Barro ${ }^{2} \&$ Gabriela Molina $^{2}$ \\ 1. Instituto de Ecología, A.C., Xalapa; anneryserrano@gmail.com, miguel.moron@inecol.mx \\ 2. Facultad de Biología, Universidad de la Habana, Cuba; abarro@fbio.uh.cu, gabybio382@gmail.com
}

\author{
Recibido 16-II-2016. Corregido 11-X-2016. Aceptado 10-XI-2016.
}

\begin{abstract}
Comparison between Phyllophaga (Coleoptera: Melolonthidae) assemblages in Western Cuba. The Cuban archipelago has 79 Phyllophaga species with a $98.6 \%$ of endemism, which makes the group ecologically and economically important, as some species are classified as crop pests. The aim of this study was to determine the relationship between the composition and structure of the Phyllophaga assemblages with their habitat type, considering both plant structure and level of human habitat disturbance. A total of 17 locations were sampled in Western Cuba during the rainy seasons of the period from 2011 to 2015; these differed in habitat types (forests and agroecosystems). Samplings followed standard methods and were made once with a light trap screen in each locality. A total of 1153 individuals of 24 Phyllophaga species were collected. The total abundance of collected individuals varied between 10 and 306, and species richness between two and nine species. The most abundant species was Phyllophaga dissimilis (Chevrolat) which also had the highest frequency of occurrence. Generally, less anthropized sites reflected richer and equitative communities; although these characteristics appeared in two sites that showed intermediate degree of disturbance. The non-metric multidimensional scaling showed that the most similar samples were those of the very anthropized sites; this similarity was given mainly by the abundance of Phyllophaga dissimilis, P. insualepinorum and P. puberula. Besides, the geographical distance was not related to the similarity of these assemblages. Rev. Biol. Trop. 65 (1): 351-363. Epub 2017 March 01.
\end{abstract}

Key words: Phyllophaga, communities, composition, abundance, bioindicator.

Los escarabaeoideos son uno de los grupos más conocidos de Coleoptera. Entre ellos, se ubica la subfamilia Melolonthinae, la cual está constituida en Cuba por dos géneros: Anoplosiagum Blanchard y Phyllophaga Harris, 1826 (Peck, 2001). Éste último se encuentra representado solamente por el subgénero Cnemarachis Saylor (Coleoptera: Scarabaeoidea: Melolonthidae: Melolonthinae) el cual es endémico del Caribe (Woodruff \& Sanderson, 2004; Evans \& Smith, 2009). Los adultos, pueden ser plagas que dañan el follaje de numerosas plantas frutales, forrajes y de ornatos. Las larvas tienen aún mayor importancia económica, puesto que se alimentan de las raíces y en ocasiones causan su deterioro (Morón, 1994b). Sin embargo, en Cuba no se conoce la intensidad de daño que estas especies causan a los cultivos, y la mayor parte de los estudios disponibles sobre el género, se enfocan en las descripciones taxonómicas. En la actualidad, están registradas 79 especies de Phyllophaga para el archipiélago cubano, con un $98.6 \%$ de endemismo (Smith \& Paulsen, 2015), lo cual le confiere al grupo una importancia adicional en la región.

Este género está ampliamente distribuido en toda la isla, aunque se ha considerado que la mayoría de sus especies son muy localizadas (García-Vidal, 1975). De hecho, este ha llegado a ser un criterio de peso en la descripción de nuevas especies (García-Vidal, 1984). 
Para el occidente cubano, están descritas 35 especies (García-Vidal,1987,1988; Muslera \& Fernández, 1998) lo cual representa el $41.7 \%$ de las existentes en la isla.

Algunos autores han propuesto considerar a las especies de Phyllophaga y otros Melolonthidae como elementos indicadores ecológicos y zoogeográficos, ya que se ha observado la gran importancia ecológica que tienen como consumidores primarios y como fuente de alimento para especies mayores, lo cual deriva primordialmente de su abundancia y gran capacidad de adaptación (Morón, 2001, 2006, 2014; Yanes-Gómez \& Morón, 2010).

Se ha observado que el tipo de vegetación influye directamente en la riqueza de Scarabaeoidea (García-Vidal, 1975; Morón \& Zaragoza, 1976; Morón, 1994a, 1994b; Morón-Ríos \& Morón, 2001; Delgado \& Márquez, 2006). Por otra parte, el tipo de vegetación varía de acuerdo con la precipitación y la temperatura, cuyos valores se consideran también determinantes en la diversidad de Melolonthidae, observándose mayor abundancia y riqueza de especies durante los meses con lluvia intensa y temperatura media mensual alta (Morón \& Deloya, 1991; Morón-Ríos \& Morón, 2001; Delgado, CastroRamírez, Morón, \& Ruiz-Montoya, 2012).

El objetivo de este trabajo es determinar la relación entre la composición y estructura de los ensambles de Phyllophaga y el tipo de hábitat (en cuanto a formación vegetal y grado de alteración). El estudio de la ecología de las especies del género es tarea fundamental para la biología de la conservación, y el diseño de planes de manejo agrícolas y forestales.

\section{MATERIALES Y MÉTODOS}

Área de estudio: Los muestreos fueron realizados entre mayo y agosto (ya que los adultos emergen cuando comienzan las lluvias) desde 2011 al 2015 en el occidente de Cuba. Fueron muestreadas un total de 26 localidades en el occidente de Cuba, seleccionándolas a priori de manera que fueran representativas de esta región del país. Sin embargo, por el tipo de método empleado, solo se tuvieron en cuenta aquellas donde fueron recolectados más de 10 ejemplares de Phyllophaga, por lo que se resumieron a 17 sitios. Las 17 localidades analizadas (Fig. 1), difieren en cuanto al tipo de hábitat y grado de alteración según lo mostrado en el cuadro 1. El grado de alteración fue asignado cualitativamente teniendo en cuenta el tipo de uso de suelo y la cercanía a asentamientos humanos: tres fue dado a zonas con cultivos, áreas urbanas y suburbanas; dos, a zonas con vegetación secundaria o parche de vegetación remanente cercano a asentamientos humanos y uno a las áreas de colecta lejanas de asentamientos humanos y con vegetación natural.

En esta región están representadas una amplia variedad de tipos de vegetación y predominan las zonas antropizadas con cultivos o poblados y los bosques secundarios, aunque quedan algunos remanentes del bosque original, sobre todo a mayor altitud y dentro de áreas protegidas. El tipo de formación vegetal de cuabal o serpentina se caracteriza por estar constituida de plantas xerófitas, se relaciona con afloramientos de rocas ultramáficas. Los cuabales están enclavados en suelos delgados a muy poco profundos, pedregosos y poco desarrollados. Son suelos ligeramente ácidos donde la materia orgánica se encuentra superficialmente acumulada en las oquedades de las rocas y consta de hojas y otras partes vegetales muy secas, que reciben mucho sol dada la poca sombra proporcionada por la vegetación al suelo (Capote \& Berazaín, 1984; Borhidi, 1996). También, presentan un alto contenido de magnesio, níquel, cromo y cobalto (Bécquer, González, Berazaín, \& Gutiérrez, 2003). Los cuabales, conforman en la isla áreas de gran endemismo vegetal que varía, según la localidad de un 27 a un $98 \%$ en dependencia de la región geográfica y del estado de conservación de los mismos (Areces, González-Torre, \& Berazaín, 2003).

El Complejo de vegetación de mogote, se encuentra sobre elevaciones cársicas. Esta formación se caracteriza por la presencia de bosques siempreverdes y/o semideciduos, según la exposición, el sustrato, las variantes del relieve y la altura de cada mogote. 


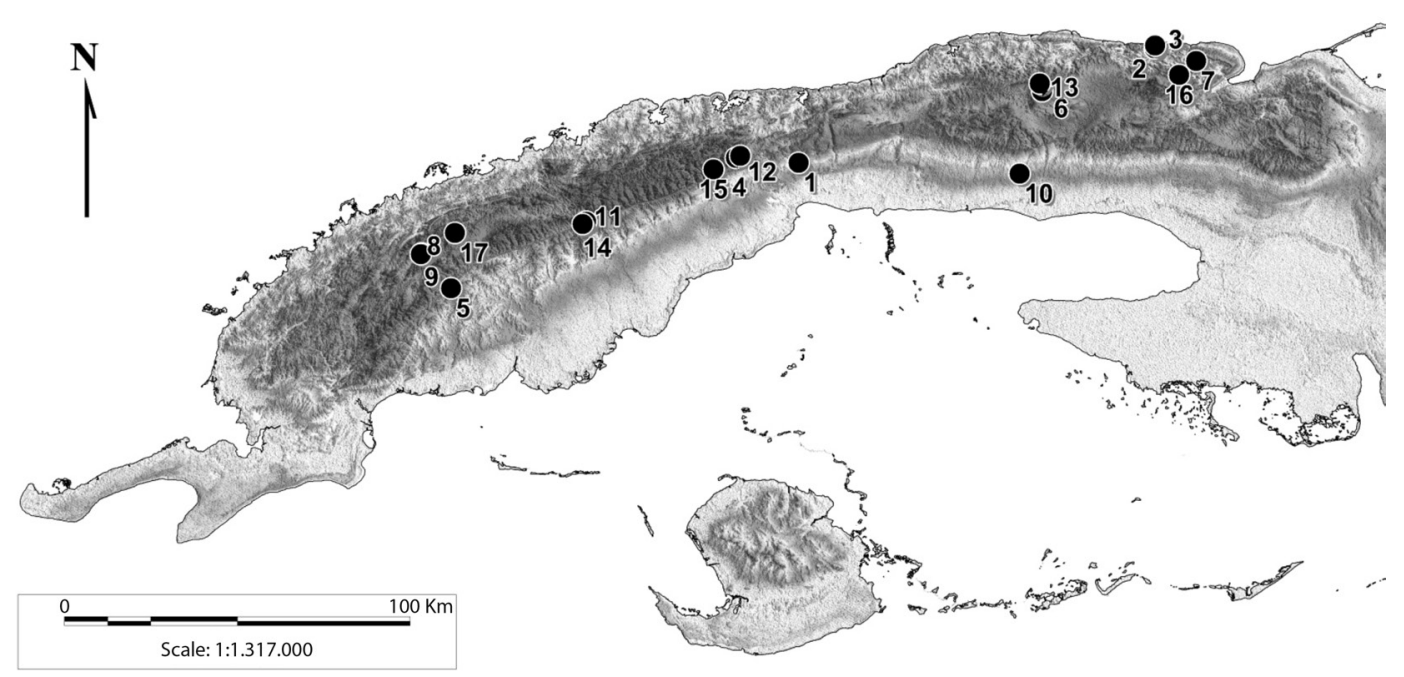

Fig. 1. Ubicación geográfica de 17 localidades muestreadas con trampas de luz (250W) en el occidente de Cuba en las que se registraron más de 10 individuos de Phyllophaga. 1: Artemisa, 2: Canasí, bosque, 3: Canasí, uveral , 4: El Salón, 5: La Guabina, 6: La Jaula, 7: Lomas de Galindo, 8: Maravillas de Viñales, bosque, 9: Maravillas de Viñales, pastizal, 10: Melena del Sur, 11: Mil Cumbres, 12: Río San Juan, 13: Reparto Siboney, HAB, 14: Sierra la Güira, 15: Soroa, 16: Tres Ceibas de Clavellinas, 17: Dos Hermanas Viñales.

Fig. 1. Location of 17 sities sampled with light traps (250W) in western Cuba where more than 10 individuals of Phyllophaga were recorded 1: Artemisa, 2: Canasí, bosque, 3: Canasí, uveral , 4: El Salón, 5: La Guabina, 6: La Jaula, 7: Lomas de Galindo, 8: Maravillas de Viñales, bosque, 9: Maravillas de Viñales, pastizal, 10: Melena del Sur, 11: Mil Cumbres, 12: Río San Juan, 13: Reparto Siboney, HAB, 14: Sierra la Güira, 15: Soroa, 16: Tres Ceibas de Clavellinas, 17: Dos Hermanas Viñales.

En la cima de las elevaciones se destacan matorrales con especies emergentes (Oviedo et al., 2006). Este tipo de formación vegetal se destaca por albergar un gran endemismo a nivel florístico (Oviedo et al., 2006) y algunos grupos de la fauna como moluscos terrestres (Hernández \& Reyes, 2013).

Dentro del tipo de formación vegetal de cultivo de importancia económica se incluyeron varios tipos tales como el tabaco, maíz, caña de azúcar, plátano, entre otros. En general, estos se establecen en valles con suelos ricos y profundos. Están bien irrigados durante todo el año y el laboreo que se realiza en ellos es de tipo artesanal.

\section{Método utilizado y análisis de datos:} Todos los individuos, excepto los de Artemisa, fueron recolectados durante dos noches, desde el ocaso (aproximadamente 19:00 horas) hasta las 2:00 horas del siguiente día mediante el montaje de una trampa de luz tipo pantalla (McFarland, 1966), con un generador de corriente y un bombillo de luz blanca de 250 W. Para la muestra se consideraron los individuos encontrados en ambas caras de la sábana, la superficie que quedó sobre el suelo, y los encontrados debajo del doblez de ésta. En la localidad de Artemisa, la recolecta fue realizada bajo los bombillos de luz blanca de $250 \mathrm{~W}$ del alumbrado público en las afueras de la ciudad.

Los especímenes recolectados fueron conservados en alcohol al $90 \%$ para luego ser identificados en el laboratorio. La identificación se llevó a cabo teniendo en cuenta los criterios mencionados en las descripciones originales (Chapin, 1932, 1935; Saylor, 1942; Chalumeau, 1989; García-Vidal, 1975, 1978, 1984, 1987, 1988; Smith \& Paulsen, 2015).

Se construyó la curva de acumulación de especies en función de la cantidad de 
CUADRO 1

Localidades de la región occidental de Cuba seleccionadas para el estudio, tipo de hábitat y grado cualitativo de alteración asignado por los autores

TABLE 1

Sites in western of Cuba selected for the study, type of habitat and qualitative and relative degree of disturbance assigned by authors

\begin{tabular}{|c|c|c|c|c|c|}
\hline & Localidad & Tipo de hábitat & $\begin{array}{c}\text { Grado de } \\
\text { alteración (1-3) }\end{array}$ & Abundancia & $\begin{array}{c}\text { Riqueza de especies } \\
\text { observada }\end{array}$ \\
\hline 1 & Artemisa & Ecotono pueblo-cultivo & 3 & 29 & 3 \\
\hline 2 & Canasí, bosque & Bosque semideciduo & 1 & 10 & 5 \\
\hline 3 & Canasí, uveral & Uveral & 2 & 10 & 2 \\
\hline 4 & El Salón & Bosque siempreverde & 1 & 11 & 4 \\
\hline 5 & La Guabina & Pastizal-cultivo & 2 & 100 & 5 \\
\hline 6 & La Jaula & Vegetación de mogote & 2 & 11 & 5 \\
\hline 7 & Lomas de Galindo & Cuabal & 1 & 48 & 4 \\
\hline 8 & Maravillas de Viñales, bosque & Bosque semideciduo & 1 & 40 & 6 \\
\hline 9 & Maravillas de Viñales, pastizal & Pastizal-bosque semideciduo & 2 & 21 & 4 \\
\hline 10 & Melena del Sur & Ecotono pueblo-cultivo & 3 & 127 & 5 \\
\hline 11 & Mil Cumbres & Ecotono pastizal-bosque siempreverde & 2 & 90 & 9 \\
\hline 12 & Rio San Juan & Ecotono cultivo-bosque semideciduo & 2 & 306 & 7 \\
\hline 13 & Reparto Siboney, La Habana & Ciudad & 3 & 146 & 4 \\
\hline 14 & Sierra la Güira & Bosque semideciduo & 1 & 64 & 4 \\
\hline 15 & Soroa & Bosque siempreverde & 1 & 68 & 9 \\
\hline 16 & Tres Ceibas de Clavellinas & Cuabal & 2 & 58 & 8 \\
\hline 17 & Dos Hermanas, Viñales & Cultivo-vegetación de mogote & 2 & 14 & 3 \\
\hline
\end{tabular}

localidades de muestreo. Para ello se empleó el estimador de riqueza de Mao Tau, Chao1 y Boostrap con 999 permutaciones en el programa EstimateS 8.2.0 (Colwell, 2006), a esta curva se le ajustó el modelo de Clench y a partir del modelo se calculó el número de especies teóricas para la región, la efectividad del muestreo y el porcentaje de las especies teóricas registradas (Jiménez-Valverde, 2003).

Se elaboraron curvas de rango de abundancia para cada localidad con el logaritmo decimal de la abundancia proporcional. Se realizó un análisis de similitud entre muestras (SIMPER) para lo cual se construyeron dos matrices de similitud: con el índice de BrayCurtis (para datos de abundancia absoluta y abundancia relativa) y de Sorensen, con datos de presencia/ausencia. El análisis SIMPER permite conocer cuáles variables (especies) contribuyen más o menos a la similitud/disimilitud dentro/entre grupos. Para ello fue empleado el programa Primer6 (2006). La matriz de similitud de Bray-Curtis se correlacionó, mediante una prueba de Mantel, con la de distancia entre localidades para saber si existía relación entre estas dos variables.

Se realizó un escalado multidimensional no métrico (MDS) con la abundancia absoluta y la abundancia proporcional de cada especie por localidad. Los valores de los ejes obtenidos en el ordenamiento de las muestras, fueron extraídos y comparados entre grupo de localidades de diferente grado de alteración usando un análisis de varianza con corrección de Welch para muestras sin homogeneidad de varianza.

\section{RESULTADOS}

Se recolectaron 1153 ejemplares de 24 especies de Phyllophaga, con tres nuevos registros para la región occidental. Todas las especies, excepto P. patruelis, son endémicas 
de Cuba. La abundancia total de individuos recolectados varió entre 10 (ya que este fue el corte mínimo de abundancia de una localidad para incluirla en el análisis) en Canasí, y 306 en el Río San Juan, donde se colectó la mayor cantidad de ejemplares (Cuadro 1).

La especie Phyllophaga dissimilis (Chevrolat) fue la más abundante y la de mayor frecuencia de ocurrencia. Otras especies, tuvieron alta abundancia, que coincidió, aunque no de manera directa, con la alta representatividad en las muestras (Cuadro 2). Cinco de las especies colectadas aún no están identificadas y probablemente constituyan nuevos registros para Cuba.
La curva de acumulación de especies (Fig. 2), realizada con la Sobs Mao Tau con 999 iteraciones no se saturó, lo cual significa que los muestreos, según este estimador, y otros como Chaol y Bootstrap, son insuficientes para registrar la totalidad de las especies presentes en la zona. Los datos mostraron un buen ajuste al modelo de Clench $\left(\mathrm{R}^{2}=0.989\right)$ y basado en este modelo, se puede decir que las especies reportadas sólo conforman el 73 $\%$ del número total teórico de especies para la región (58). Según este modelo, con un $95 \%$ de intervalos de confianza, harían falta un total de 137 muestreos para registrarlas a todas y 38 muestreos para registrar $80 \%$ de ellas.

\section{CUADRO 2}

Frecuencia de ocurrencia y abundancia proporcional con respecto al total de individuos de Phyllophaga capturados con trampa de luz en 17 localidades del occidente de Cuba de entre mayo y agosto de 2011-2015. NR: Nuevo registro para el occidente de Cuba

TABLE 2

Frequency of occurrence and proportional abundance of the total of Phyllophaga individuals captured with a light trap in 17 sites in Western Cuba between May and August of 2011-2015. NR: New register for Western Cuba

\begin{tabular}{|c|c|c|}
\hline Especie & Frecuencia de ocurrencia (\%) & Abundancia proporcional (\%) \\
\hline P. dissimilis (Chevrolat) 1865 & 76.47 & 30.01 \\
\hline P. vermiculata Chapin 1932 & 23.53 & 23.24 \\
\hline P. trinitariensis Garcia Vidal 1987 (NR) & 52.94 & 10.67 \\
\hline P. tuberculifrons (Chevrolat) 1865 & 35.29 & 10.49 \\
\hline P. insulaepinorum Garcia Vidal 1978 & 64.71 & 8.67 \\
\hline P. puberula (Jacquelin du Val) 1856 & 47.06 & 7.63 \\
\hline P. analis (Burmeister) 1855 & 47.06 & 2.17 \\
\hline P. bimammifrons Saylor 1940 & 11.76 & 1.47 \\
\hline P. dubitatus Garcia Vidal 1978 & 23.53 & 1.39 \\
\hline P. crenaticollis (Blanchard) 1850 & 23.53 & 1.21 \\
\hline P. bruneri Chapin 1932 & 23.53 & 0.52 \\
\hline P. alquizara Chapin 1932 & 11.76 & 0.35 \\
\hline P. aeruginosa (Burmeister) 1855 & 5.88 & 0.35 \\
\hline Phyllophaga sp. 2 & 5.88 & 0.26 \\
\hline P. expansa Chapin 1932 (NR) & 5.88 & 0.17 \\
\hline P. microsoma Chapin 1932 & 5.88 & 0.17 \\
\hline P. patruelis (Chevrolat) 1865 & 5.88 & 0.17 \\
\hline P. schwarsi Chapin 1932 (NR) & 5.88 & 0.17 \\
\hline P. signaticollis (Burmeister) 1855 & 5.88 & 0.17 \\
\hline Phyllophaga sp. 1 & 5.88 & 0.17 \\
\hline Phyllophaga sp. 4 & 5.88 & 0.17 \\
\hline Phyllophaga sp. 5 & 5.88 & 0.17 \\
\hline P. santaclarae Chapin 1932 & 5.88 & 0.09 \\
\hline Phyllophaga sp. 3 & 5.88 & 0.09 \\
\hline
\end{tabular}




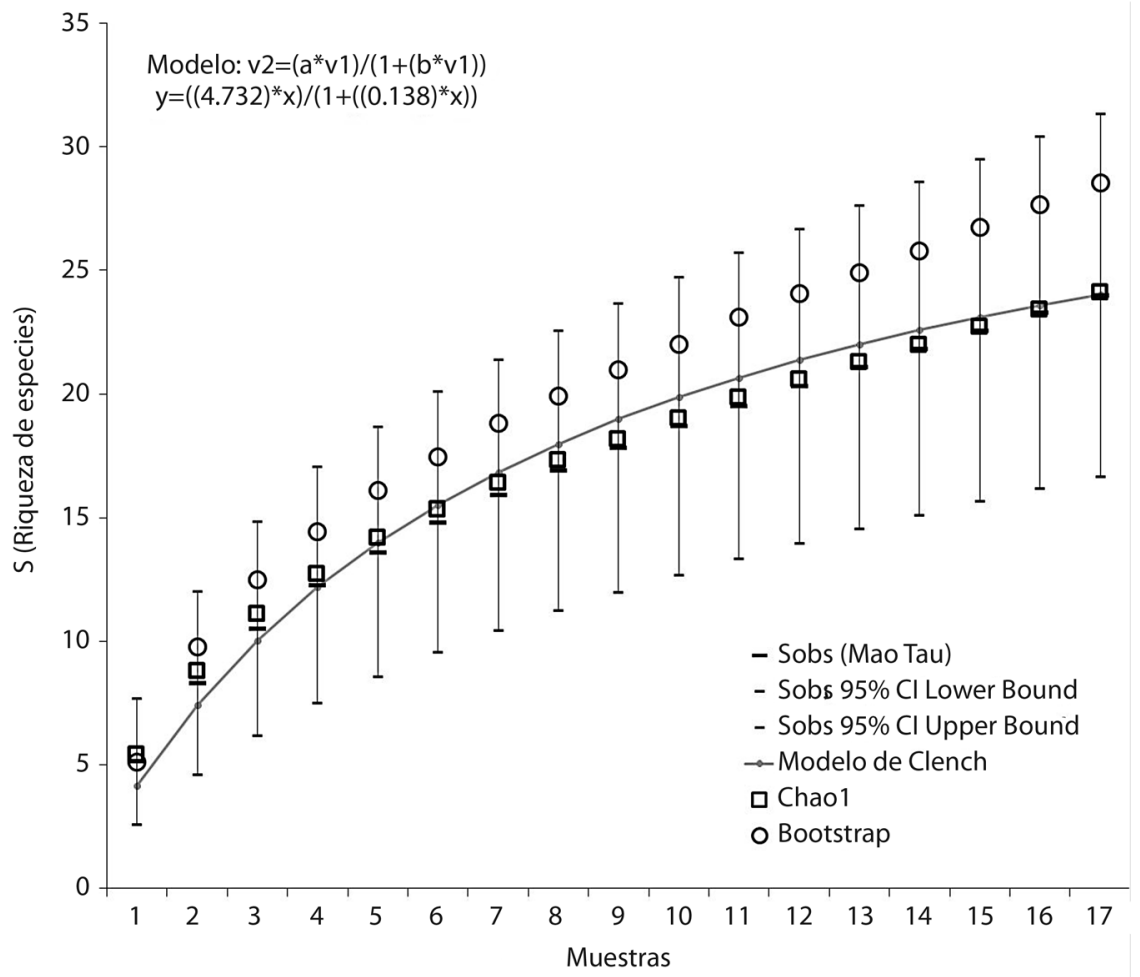

Fig. 2. Curva de acumulación de especies de Phyllophaga construida a partir de 17 localidades, del occidente de Cuba, según el estimador de riqueza de especies de MaoTau (999 iteraciones) con los intervalos de confianza $95 \%$ y ajuste al modelo propuesto por Clench.

Fig. 2. Species accumulation curve of Phyllophaga from 17 sites, in Western Cuba, according to the estimator of species richness MaoTau (999 iterations) with $95 \%$ confidence intervals and adjustted to a model proposed by Clench.

La riqueza de especies por localidad varió de nueve a dos. Siendo las más ricas en cuanto al número de especies Soroa, Mil Cumbres, Tres Ceibas de Clavellinas y el Rio San Juan, la mayor parte de ellas asociadas a formaciones vegetales boscosas. Las menos ricas fueron el uveral de Canasí, Artemisa y Dos Hermanas en Viñales (Fig. 3).

Las curvas de rango abundancia por localidad (Fig. 3) mostraron que éstas se diferencian también en la equitatividad. De manera general, las localidades menos perturbadas (2: Canasí, bosque, 4: El Salón, 8: Maravillas de Viñales bosque, 15: Soroa) reflejaron comunidades más equitativas, aunque esta característica también la compartieron Mogote La Jaula (6), Mil Cumbres (11) y Tres Ceibas de Clavellinas (16) con grado de alteración intermedio.
Las comunidades menos equitativas fueron las tres más perturbadas, tres de perturbacion intermedia y una de poco grado de alteración: Sierra la Güira (14).

El escalado multidimensional no métrico, en su representación bidimensional, reflejó que las muestras más semejantes entre sí son las pertenecientes a sitios con grado 3 de antropización (Fig. 4). Se observó una ligera tendencia de las localidades grado 3 y 1 a formar dos grupos, aunque no están muy bien definidos por la alta variabilidad entre el resto de las muestras. Existen diferencias en cuanto a las medias para los valores de cada uno de los ejes. Estas diferencias estuvieron dadas, en ambos casos entre el grupo de localidades poco antropizadas y las muy antropizadas (Cuadro 3). 

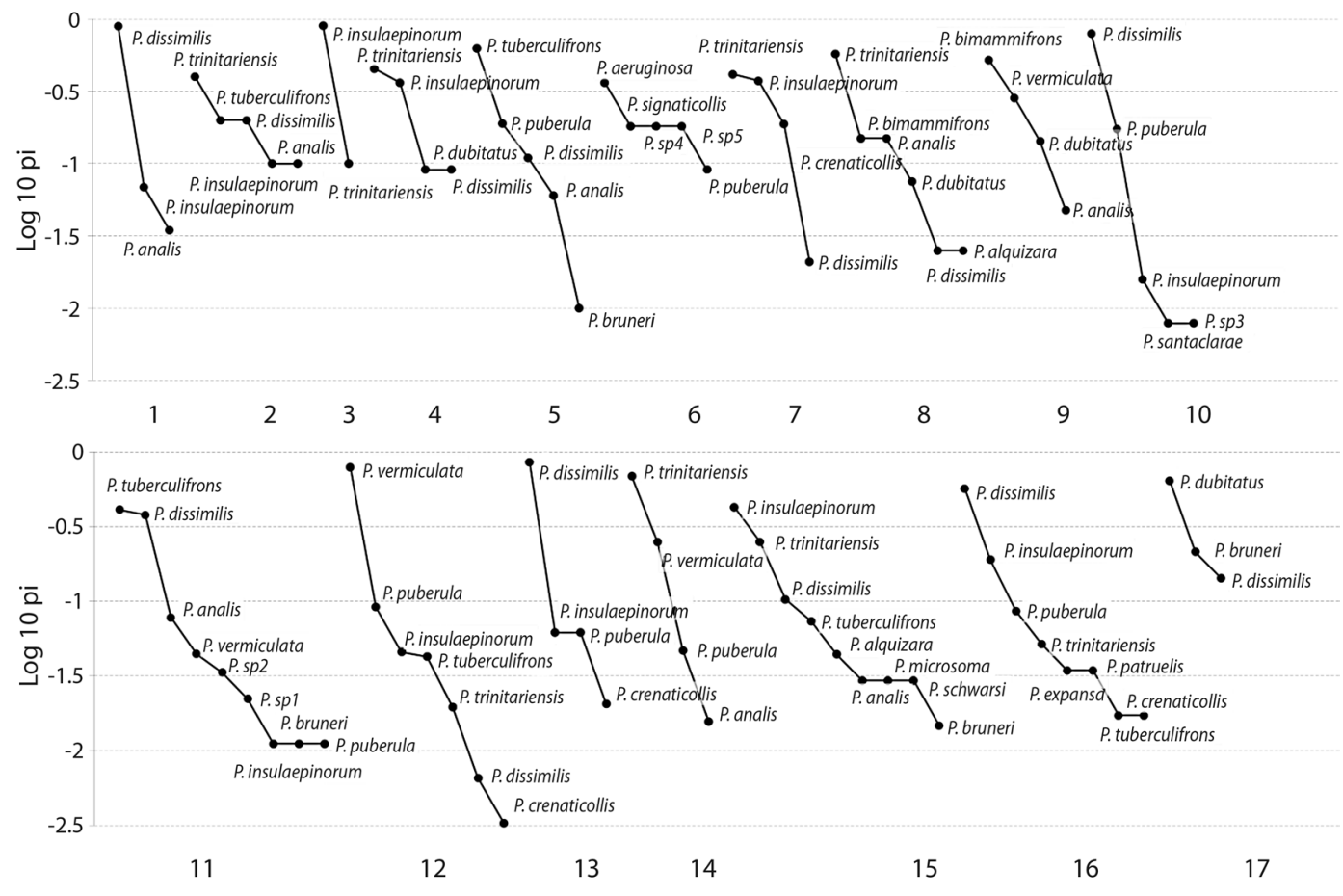

Fig. 3. Curvas de rango abundancia de las especies de Phyllophaga registradas en 17 localidades del occidente de Cuba. 1: Artemisa, 2: Canasí, bosque, 3: Canasí, uveral, 4: El Salón, 5: La Guabina, 6: La Jaula, 7: Lomas de Galindo, 8: Maravillas de Viñales, bosque, 9: Maravillas de Viñales, pastizal, 10: Melena del Sur, 11: Mil Cumbres, 12: Río San Juan, 13: Reparto Siboney, 14: Sierra la Güira, 15: Soroa, 16: Tres Ceibas de Clavellinas, 17: Dos Hermanas, Viñales.

Fig. 3. Range abundance curves of Phyllophaga species registered in 17 sites in Western Cuba. 1: Artemisa, 2: Canasí, bosque, 3: Canasí, uveral, 4: El Salón, 5: La Guabina, 6: La Jaula, 7: Lomas de Galindo, 8: Maravillas de Viñales, bosque, 9: Maravillas de Viñales, pastizal, 10: Melena del Sur, 11: Mil Cumbres, 12: Río San Juan, 13: Reparto Siboney, 14: Sierra la Güira, 15: Soroa, 16: Tres Ceibas de Clavellinas, 17: Dos Hermanas, Viñales.

No existió mucha similitud entre las muestras pertenecientes al mismo grupo en cuanto al grado de alteración teniendo en cuenta la abundancia proporcional de las especies: grupo 1 (poco perturbado) $52.06 \%$, grupo 2 (alteración media): $11.63 \%$ y grupo 3 (alta alteración): $86.72 \%$. Estos resultados explican la poca agregación de las muestras pertenecientes al mismo grupo en el MDS. Sin embargo, las diferencias entre grupos son más marcadas (Cuadro 4). Phyllophaga dissimilis es la especie que más aportó a la diferencia entre localidades más antropizadas y las menos antropizadas (3 y 1), y también entre las más antropizadas y las que tienen grado intermedio ( 3 y 2 ), ya que es la especie más dominante cuando el grado de alteración fue alto. Por otra parte, P. trinitariensis, aunque está bien representada, con más del $50 \%$ de ocurrencia en las muestras, no estuvo presente en ninguna de las localidades muy antropizadas y se encontró mejor distribuida entre las poco y medianamente antropizadas.

La distancia geográfica no se relacionó con la similitud entre ellas según el índice de Bray-Curtis con la abundancia absoluta $(\mathrm{R}=$ $-0.137, \mathrm{P}=0.0641,999$ iteraciones con bootstrap) ni con la abundancia proporcional $(\mathrm{R}=$ $-0.1297, \mathrm{P}=0.0876,999$ iteraciones con bootstrap) aunque sí hay una ligera correlación de la distancia y el índice de Sorensen de presencia ausencia $(\mathrm{R}=-0.307, \mathrm{P}<0.001,999$ iteraciones con bootstrap). 

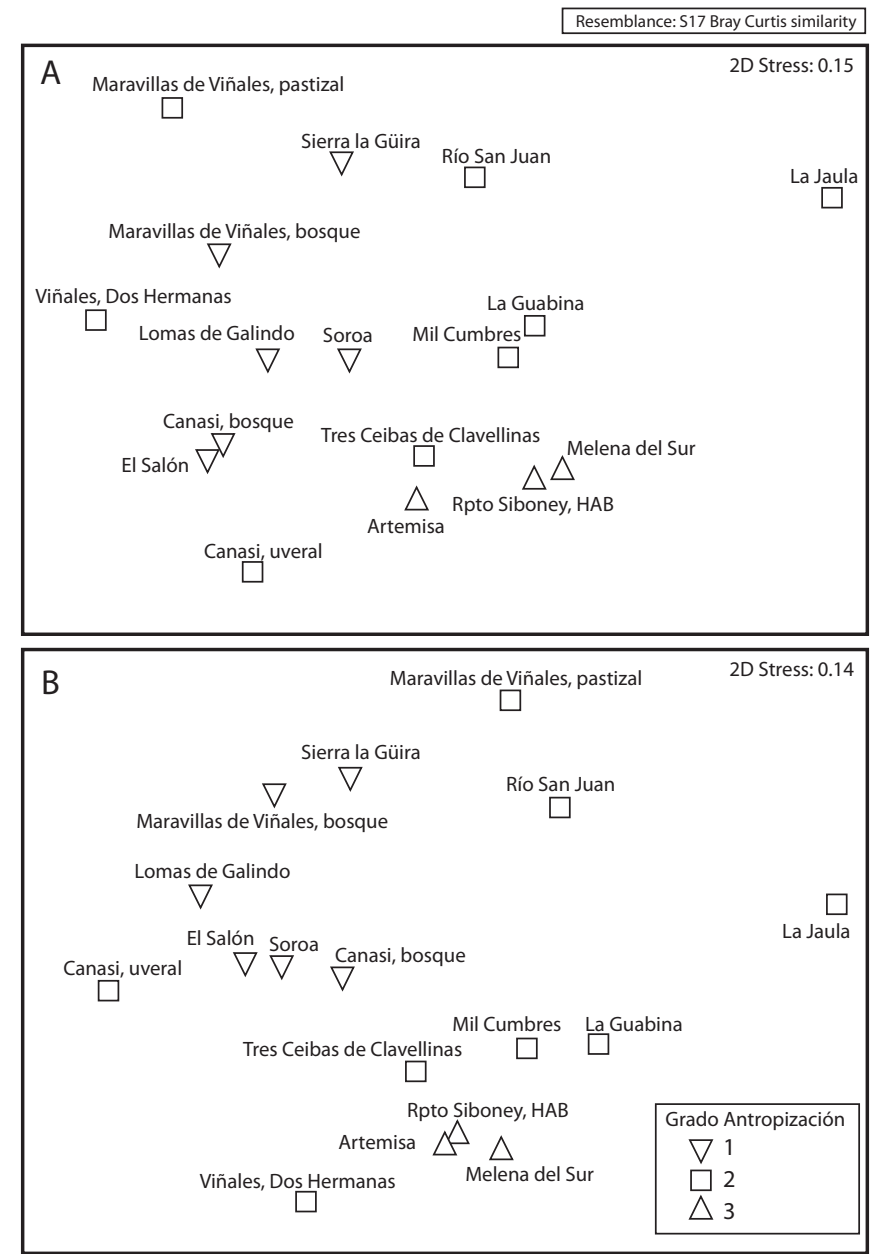

Fig. 4. Escalado Multidimensional no Métrico (MDS no métrico) entre muestras de las diferentes localidades agrupadas por el grado de alteración de cada una 1: bajo, 2: medio, 3: alto. A. MDS no métrico con los datos de abundancia absoluta para cada una de las especies. B. MDS no métrico con la abundancia proporcional de cada una de las especies por localidad. Fig. 4. Non Metric Multidimensional Scaling (Non Metric-MDS) between samples from different sites grouped by the degree of disturbance of each one 1: Low, 2: Medium, 3: High. A. Non metric-MDS with species absolute abundance data. B. Non metric-MDS with proportional abundance of each species per location.

\section{DISCUSIÓN}

Independientemente de la abundancia observada en las zonas antropizadas, la riqueza de especies es baja. Lo cual coincide con una menor heterogeneidad ambiental asociada a cultivos y zonas de la periferia urbana. La riqueza de especies de algunos grupos de la macrofauna disminuye a medida que aumentan las perturbaciones antrópicas dentro del ecosistema (Rossi \& Blanchart, 2005). Esto concuerda con lo expresado por Delgado et al. (2012), quienes concluyeron que para Melolonthidae, factores como la cobertura arbórea y herbácea, condicionan la riqueza de especies por tratarse en su mayoría de especies rizófagas, observando un incremento de la riqueza conforme la estructura vegetal se hace más compleja. Otros autores, sin embargo, registraron para el género una mayor riqueza asociada a zonas de cultivos (Rivera-Gasperín, Carrillo-Ruiz, Morón, \& Yanes-Gómez, 
CUADRO 3

Análisis de varianza realizado entre grupos con diferente grado de alteración teniendo en cuenta los resultados de los dos ejes obtenidos en el MDS

TABLE 3

Analysis of variance performed between groups with different degrees of disturbance considering the results of the two axes obtained in the MDS

\begin{tabular}{lcccc} 
& \multicolumn{4}{c}{ Análisis de varianza de resultados del MDS } \\
& \multicolumn{1}{c}{ Abundancia absoluta (A) } & Abundancia proporcional (B) \\
Ejes del MDS & Eje 1 & Eje 2 & Eje 1 & Eje 2 \\
Welch F & 10.41 & 8.658 & 19.92 & 26.16 \\
df & 7.982 & 8.203 & 9.207 & 7.953 \\
p & 0.006 & 0.0096 & $<0.001$ & $<0.001$ \\
& \multicolumn{1}{c}{ Comparación de medias entre poco y muy perturbados (1 y 3) } \\
Prueba U de Mann-Whitney & 0 & 0 & 0 & -2.1947 \\
Z & -2.1947 & & -2.1947 & 0.0266 \\
p (9999 permutaciones Bootstrap): & 0.0244 & & 0.0225 & \\
\hline
\end{tabular}

Se muestran los resultados significativos en la comparación de medias a posteriori, en este caso la probabilidad fue calculada usando el método de remuestreo bootstrap con 999 iteraciones.

Significant results are shown in subsequent comparison of means, in this case the probability was calculated using the bootstrap resampling method with 999 iterations.

2013). En este trabajo, las zonas asociadas a formaciones vegetales boscosas son las que presentan una mayor riqueza de especies (entre 6 y 9). Tres Ceibas de Clavellinas con 8 especies y vegetación predominante de serpentina es la excepción.

En un área similar, aunque continental, fueron registradas 24 morfoespecies de Phyllophaga (Castro-Ramírez, Delfín, Parra, \& Morón, 2005), riqueza similar a la encontrada por nosotros en este estudio. Otras investigaciones puntuales en escarabeidos reportan desde ninguna hasta 16 especies de Phyllopha$g a$, aunque incluyen también áreas mayores y con diferentes tipos de usos de suelo (Aragón \& Morón, 2000; Morón-Ríos \& Morón, 2001; Yanes-Gómez \& Morón, 2010; García-Atencia \& Martínez-Hernández, 2015). Estos trabajos en ocasiones registran una mayor riqueza porque complementan diferentes métodos de captura. No todas las especies de Phyllophaga son atraídas a la luz, y por tanto es necesario establecer muestreos basados en recolectas directas sistemáticas adicionales (Méndez-Aguilar, Castro-Ramírez, Barrantes, Pacheco-Flores, \& Ramírez-Salinas, 2005).
Las diferencias observadas en el número de especies en cada localidad están determinadas posiblemente por la acción conjunta del tipo de formación vegetal y el grado de alteración de cada sitio. No se descartan otros factores a nivel de paisaje, como el tipo de matriz y el grado de fragmentación que tenga el tipo de hábitat muestreado.

Igualmente, las curvas de rango abundancia muestran, en estas localidades, una alta dominancia característica de lugares con estrés ambiental o altamente perturbados, según lo reportado para Coleoptera y otros insectos en general (Brown, Pasini, Benito, Aquino, \& Fernandes, 2001).

Phyllophaga dissimilis es la especie mejor representada en términos de abundancia y distribución. Esta especie se ha registrado como plaga del cultivo de la caña de azúcar (Peck, 2001) lo cual justifica su amplia representatividad en el occidente del país, donde este cultivo es aún muy abundante a pesar que se ha visto reducido en los últimos años. Ha sido colectada hasta el oriente del país (Muslera \& Fernández, 1998), por lo tanto, su distribución es pancubana; también se registró en la Florida, 
CUADRO 4

Resultados del análisis de similitud (SIMPER) entre los tres grupos de localidades con diferente grado de alteración

TABLE 4

Results of the analysis of similarity (SIMPER) among the three groups of sites with different degrees of disturbance

Factor: Grado de alteración

Disimilitud promedio entre grupos: 88.15

\begin{tabular}{lccc}
\multicolumn{1}{c}{ Especies } & \multicolumn{2}{c}{ Abundancia proporcional promedio } & Disimilitud (\%) \\
P. dissimilis & Alterado (3) & Poco alterado (1) & 44.02 \\
P. trinitariensis & 0.85 & 0.07 & 26.32 \\
P. insulaepinorum & 0 & 0.46 & 11.04 \\
\hline
\end{tabular}

Disimilitud promedio entre grupos: 79.64

\begin{tabular}{lccc}
\multicolumn{1}{c}{ Especies } & \multicolumn{1}{c}{ Disimilitud promedio entre grupos: 79.64} & Disimilitud (\%) \\
P. dissimilis & Alterado (3) & Medio alterado (2) & 43.86 \\
P. insulaepinorum & 0.85 & 0.15 & 9.79 \\
P. vermiculata & 0.05 & 0.14 & 8.8 \\
P. tuberculifrons & 0 & 0.14 & 8.64 \\
P. dubitatus & 0 & 0.14 & 6.17
\end{tabular}

Disimilitud promedio entre grupos: 84.82

\begin{tabular}{lccc}
\multicolumn{1}{r}{ Especies } & \multicolumn{1}{c}{ Abundancia proporcional promedio } & Disimilitud (\%) \\
P. trinitariensis & Poco alterado (1) & Medio alterado (2) & 26.09 \\
P. insulaepinorum & 0.46 & 0.02 & 15.57 \\
P. vermiculata & 0.21 & 0.14 & 9.38 \\
P. dissimilis & 0.04 & 0.14 & 9.17 \\
P. tuberculifrons & 0.07 & 0.15 & 9.16 \\
P. dubitatus & 0.05 & 0.14 & 6.6 \\
\hline
\end{tabular}

El cuadro muestra el promedio de la abundancia proporcional de cada especie dentro del grupo, y la disimilitud que aporta cada una. Solo se muestran los resultados por encima del $5 \%$.

The table shows the average of proportional abundance of each species within the group, and the dissimilarity that gives each. It shows only the results above $5 \%$.

donde al parecer, se introdujo por este mismo cultivo (Peck, 2001).

Phyllophaga insulaepinorum y P. trinitariensis también son especies abundantes y frecuentes, presentes en más de la mitad de las localidades muestreadas. A diferencia de P. dissimilis ninguna de estas dos especies ha sido registrada como plaga de cultivo, pero es necesario realizar trabajo de campo para verificar sus hábitos alimentarios. Hasta el momento $P$. insulaepinorum solo había sido reportada para la Isla de la Juventud. En este estudio se comprueba que su distribución abarca también la Isla de Cuba al menos hasta la provincia de Matanzas. Por su parte P. trinitariensis, se había colectado hasta ahora solamente en el centro del país, y ahora se encontró en nueve de las localidades muestreadas. En este trabajo, se amplía la distribución de otras dos especies hasta el occidente del país: $P$. expansa y $P$. schwarsi, registradas hasta el presente en una sola localidad, y se registran posiblemente cinco nuevas especies para Cuba que permanecen sin determinar.

Según los datos teóricos de riqueza de especies y eficiencia de esfuerzo de muestreo, por el ajuste de la curva de acumulación a la curva de Clench, faltan por registrar 
34 especies para la región. Previamente, para esta región habían sido descritas 35 especies (Peck, 2001). Esto resulta en 40 especies de Phyllophaga para el occidente de la Isla de Cuba, más del $50 \%$ de las 75 especies predichas por (Woodruff \& Sanderson, 2004).

La similitud calculada entre los ensambles de los diferentes sitios y el MDS muestran que existe cierto recambio de especies entre localidades, 12 de las 24 especies registradas son exclusivas de una localidad. Esto, permite explicar el hecho de que la similitud entre localidades con el mismo grado de alteración sea baja, pues este análisis toma también en cuenta las especies únicas y compartidas, asi como su abundancia.

Se puede decir que $P$. dissimilis y $P$. insulaepinorum parecen ser especies bioindicadoras de alteración ambiental y pueden estar asociadas a los cultivos como nocivas dada la alta abundancia con la que aparecen. Cinco localidades en las que aparece $P$. dissimilis son de media y baja alteración. Para $P$. insulaepinorum de las 11 localidades donde fue observada, 4 fueron de media y baja alteración. Sin embargo, solo tiene altos valores de dominacia en aquellas localidades más perturbadas. En la localidad de Canasí bosque esto no ocurre así, posiblemene por su cercanía a una formación vegetal monotípica: Canasí uveral favorable para que se desarrollen especies oportunistas.

La poca relación entre la cercanía geográfica y la composición y estructura de los ensambles parece corroborar que las características de los ensambles de Phyllophaga dependen más de las características del hábitat para su colonización, que de su capacidad de dispersión.

Es importante destacar que la riqueza de especies observada en este trabajo para cada localidad, al igual que el resto de las características del ensamble, parten de un estudio muy puntual en cada una y por tanto preliminar. Para determinar con certeza la riqueza de especies de cada una, serán necesarios muestreos mas intensivos y la construcción de curvas de acumulación de especies a lo largo de varias temporadas de emergencia de adultos. Los muestreos deben ser más intensivos y se debe incluir la búsqueda y clasificación de larvas. Sin embargo, este trabajo constituye el primer acercamiento a este género en Cuba, desde el punto de vista ecológico.

\section{AGRADECIMIENTOS}

A Sheila González por la ayuda en el campo y en procesamiento del material. A Paul Sosa, Aryamne Serrano, Claudia Loiz y Freddy Hernández por el apoyo con los trabajos de campo. A Sergio Ibáñez.

\section{RESUMEN}

El archipiélago cubano cuenta con 79 especies de Phyllophaga (Coleoptera: Melolonthidae), con un $98.6 \%$ de endemismo, lo cual le confiere al grupo importancia desde el punto de vista de biodiversidad y económica, al ser algunas especies plagas de cultivos. El objetivo de este trabajo fue determinar la relación entre la composición y estructura de los ensambles de Phyllophaga y el tipo de hábitat (en cuanto a formación vegetal y grado de alteración humana). Durante la estación de lluvia fueron muestreadas 17 localidades del occidente de Cuba, las que difieren en cuanto al tipo de hábitat y grado de alteración. Las recolectas fueron realizadas con una trampa de luz tipo pantalla (McFarland, 1966). Se recolectaron 1153 ejemplares de 24 especies de Phyllophaga. La abundancia total de individuos recolectados varió entre 10 y 306 y la riqueza de especies entre dos y nueve especies. Phyllophaga dissimilis (Chevrolat) fue la especie más abundante y la de mayor frecuencia de ocurrencia. De manera general, las localidades menos antropizadas reflejaron comunidades más ricas y equitativas, aunque estas características también la tuvieron dos sitios que tienen grado de alteración intermedia. El escalado multidimensional no métrico, reflejó que las muestras más semejantes entre sí fueron las pertenecientes a lugares muy antropizados, esta semejanza estuvo dada principalmente por la abundancia de Phyllophaga dissimilis, P. insulaepinorum y P. puberula. La distancia geográfica no se relacionó con la similitud de los ensambles.

Palabras clave: Phyllophaga, comunidades, composición, abundancia, bioindicador.

\section{REFERENCIAS}

Aragón, A. \& Morón, M. A. (2000). Los coleópteros Melolonthidae asociados a la rizosfera de la caña de azúcar en Chietla, Puebla, México. Folia Entomológica Mexicana, 79-94. 
Areces, F., González-Torre, L., \& Berazaín, R. (2003). Diversidad de plantas (Spermatophyta) en distritos fitogeográficos de Cuba ¿Sustentan los distritos ultramáficos la mayor diversidad? En R. S. Boyd, A. Baker, \& J. Procto (Eds.), Rocas ultramáficas: sus suelos, vegetación y fauna (pp. 347). Reino Unido: Science Review.

Bécquer, E., González, L., Berazaín, R., \& Gutiérrez, J. (2003). Flora y vegetación del afloramiento ultramáfico de San Felipe, Jatibonico, Cuba Central. En R. S. Boyd, A. Baker, \& J. Procto (Eds.), Rocas ultramáficas: sus suelos, vegetación y fauna (pp. 347). Reino Unido: Science Review.

Borhidi, A. (1996). Phytogeography and vegetation ecology of Cuba (Segunda ed.). Budapest, Hungría: Akadémiai Kiadó.

Brown, G. G., Pasini, A., Benito, N. P., Aquino, A. M., \& Fernandes, M. E. (2001). Diversidad y rol funcional de la macrofauna edáfica en ecosistemas tropicales mexicanos. Acta Zoológica Mexicana (nueva serie) (Número especial 1), 79-110.

Capote, R. P. \& Berazaín, R. (1984). Clasificación de las formaciones vegetales de Cuba. Revista del Jardín Botánico Nacional, 5(2), 27-75.

Castro-Ramírez, A. E., Delfín, H., Parra, V., \& Morón, M. A. (2005). Fauna de melolóntidos (Coleoptera: Scarabaeoidea) asociados al maíz (Zea Mays L.) en los altos de Chiapas, México. Folia Entomológica Mexicana, 44, 339-365.

Chalumeau, F. (1989). Phyllophaga Harris 1826 des Antilles (Melolonthinae): espece nouvelle, désgnation de lectotypes et synonymies (Coleoptera, Scarabaeidae). Nouvelle Revue d'Entomologie (Nouvelle Série), 6(4), 333-340.

Chapin, A. E. (1932). Revision of the pleurostict Scarabaeidae of Cuba and the Isle of Pine. Annals Entomological Society of America, XXV(1), 173-209.

Chapin, A. E. (1935). New cuban pleurostict Scarabaeidae. Memorias de la Sociedad Cubana de Historia Natural, 8(2), 67-75.

Colwell, R. K. (2006). EstimateS. Statistical estimation of species richness and shared species from samples. Versión 8.

Delgado, J. M., Castro-Ramírez, A. E., Morón, M. A., \& Ruiz-Montoya, L. (2012). Diversidad de Scarabaeoidea (Coleoptera) en las principales condiciones de hábitat de Montebello, Chiapas, México. Acta Zoológica Mexicana (nueva serie), 28, 185-210.

Delgado, L. \& Márquez, J. (2006). Estado del conocimiento y conservación de los coleópteros Scarabaeoidea (Insecta) del Estado de Hidalgo, México. Acta Zoológica Mexicana (nueva serie), 22(2), 57-108.
Evans, A. V., \& Smith, A. B. (2009). An electronic checklist of the New World chafers (Coleoptera:Scara baeidae:Melolonthinae).Version3.,from http://www. museum.unl.edu/research/entomology/SSSA/nwmelos.htm

García-Atencia, S. \& Martínez-Hernández, N. (2015). Escarabajos fitófagos (Coleoptera: Scarabaeidae) del departamento del Atlántico, Colombia. Acta Zoológica Mexicana (nueva serie), 31, 89-96.

García-Vidal, M. (1975). Compilación de los principales aspectos relativos al género Phyllophaga Harris. Agrotecnia de Cuba, 7(1-2), 17-22.

García-Vidal, M. (1978). El género Phyllophaga Harris, 1826 (Coleoptera: Scarabaeidae) en Cuba. Descripción de cinco nuevas especies. Poeyana, (182), 1-14.

García-Vidal, M. (1984). The genus Phyllophaga Harris, 1826 (Coleoptera: Scarabaeidae) in Cuba: II. Descriptions of eleven new species and illustrations of female genitalia of twelve other Phyllophaga. The Pan-Pacific Entomologist, 60(4), 313-325.

García-Vidal, M. (1987). The genus Phyllophaga Harris, 1826 (Coleoptera: Scarabaeidae) in Cuba: IV. Descriptions of six new. Journal of the New York Entomological Society, 95(2), 302-306.

García-Vidal, M. (1988). The genus Phyllophaga Harris, 1826 (Coleoptera: Scarabaeidae) in Cuba: III. Descriptions of six new species and the male $P h$. cardini Chapin. The Pan-Pacific Entomologist, 60(2), 101-106.

Hernández , M. \& Reyes, B. (2013). Composición y estructura en agregaciones de moluscos terrestres en el Complejo de vegetación de mogote, Escaleras de Jaruco, Cuba. Revista de Biología Tropical, 61(4), 1769-1783.

Jiménez-Valverde, A. (2003). Las curvas de acumulación de especies y la necesidad de evaluar la calidad de los inventarios biológicos. Revista Ibérica de Aracnología, 8, 151-161.

McFarland, N. (1966). A moth sheet for atracting and retaining live specimens without the use of a trap or tent-enclosure. Journal of Research on the Lepidoptera, 5(1), 29-36.

Méndez-Aguilar, M. D., Castro-Ramírez, A. E., Barrantes, R. A., Pacheco-Flores, C., \& Ramírez-Salinas, C. (2005). Eficacia de dos tipos de recolecta para registrar la diversidad de melolóntidos nocturnos (Coleoptera: Scarabaeoidea). Acta Zoológica Mexicana (nueva serie), 21(3), 100-124.

Morón, M. A. (1994a). Fauna de coleoptera Lamellicornia en las Montañas del noreste de Hidalgo, México. Acta Zoológica Mexicana (nueva serie), 63, 7-59. 
Morón, M. A. (1994b). La diversidad genética de los coleopteros Melolonthidae en México. Acta Zoológica Mexicana (nueva serie), 61, 7-19.

Morón, M. A. (2001). Larvas de escarabajos del suelo en México (Coleoptera: Melolonthidae). Acta Zoológica Mexicana (nueva serie), 1, 111-130.

Morón, M. A. (2006). Composición de comunidades de Phyllophaga en México (Coleoptera: Melolonthidae: Melolonthinae). In A. Castro-Ramírez, M. A. Morón, \& A. Aragón (Eds.), Diversidad, importancia y manejo de Escarabajos edaficolas (pp. 11-36). Chiapas, México: ECOSUR, PRODUCE y BUAP.

Morón, M. A. (2014). Los escarabajos lamelicornios como indicadores ecológicos y biogeográficos. En C. A. González-Zuarth, A. Vallarino, J. C. Pérez-Jiménez, \& A. M. Low-Pfeng (Eds.), Bioidicadores: guardianes de nuestro futuro ambiental (pp. 307-324). México, DF.: El Colegio de la Frontera Sur, Instituto Nacional de Ecología y Cambio Climático.

Morón, M. A. \& Deloya, C. (1991). Los coleopteros Lamelicornios de la Reserva de la Biosfera "La Michilia" Durango, MéxicoMoron y Deloya.pdf. Folia Entomológica Mexicana, 209-283.

Morón, M. A. \& Zaragoza, S. (1976). Coleópteros Melolonthidae y Scarabaeidae de Villa de Allende, Estado de México. Anales del Instituto de Biología, Universidad Nacional Autónoma de México, Serie Zoología, 47(2), 83-118.

Morón-Ríos, A. \& Morón, M. A. (2001). La fauna de Coleoptera Melolonthidae de La Reserva de La Biósfera "El Triunfo", Chiapas, México. Acta Zoológica Mexicana (nueva serie), 1-25.

Muslera, L. \& Fernández, I. (1998). Estado actual del conocimiento del Género Phyllophaga (Coleoptera, Scarabaeidae) en Cuba. Poeyana, 461, 1-22.

Oviedo, R. P., Ventosa, I. R., Vale, A. G., Loriga, J. P., Núñez, R. A., Rodríguez, A. G., \& Rodríguez, A. (2006). Elementos generales de la naturaleza en el Área Protegida Escalera de Jaruco-Loma el Cheche
(Informe para la Empresa Nacional para la Protección de la Flora y la Fauna).

Peck, S. B. (2001). Arthropods of Florida A Checklist of the Beetles of Cuba with Data on Distributions and Bionomics (Insecta: Coleoptera). Arthropods of Florida and Neighboring Land Areas (Vol. 18., 249).

Primer6 (2006): PRIMER 6 for Windows. Plymouth Routines In Multivariate Ecological Research. PRIMER-E Ltd6 Hedingham Gardens. Roborough. Plymouth. PL6 7DX. United Kingdom.

Rivera-Gasperín, S., Carrillo-Ruiz, H., Morón, M. A., \& Yanes-Gómez, G. (2013). Fauna de Coleoptera Melolonthidae (Scarabaeoidea) en EL Rancho Canaletas, Paso del Macho, Veracruz, México. Acta Zoológica Mexicana (nueva serie), 29, 194-208.

Rossi, J. P., \& Blanchart, E. (2005). Seasonal and land-use induced variation of the composition in the Wewtern Ghats, southern India. Soil Biology and Biochemistry, 37, 1093-1104.

Saylor, L. (1942). Notes on beetles related to Phyllophaga Harris with descriptions of new genera and subgenera. Proceedings of the United States National Museum, 92(3145), 157-165.

Smith, A. B., \& Paulsen, M. J. (2015). Ten New Species of Phyllophaga Harris (Coleoptera: Scarabaeidae: Melolonthinae) from Cuba. The Coleopterists Bulletin, 69(3), 435-452.

Woodruff, R. E., \& Sanderson, M. W. (2004). Revision of the Phyllophaga of Hispaniola (Coleoptera: Scarabaeidae: Melolonthinae). Insecta Mundi, 18(1-4), 1-112.

Yanes-Gómez, G., \& Morón, M. A. (2010). Fauna de coleópteros scarabaeoidea de Santo Domingo Huehuetlán, Puebla, México. su potencial como indicadores ecológicos. Acta Zoológica Mexicana (nueva serie), 26, 123-145. 
